\title{
Glioneuronal tumors: clinicopathological findings and treatment options
}

Lidia Gatto*,1, Enrico Franceschi', Vincenzo Di Nunno1, Chiara Tomasello², Stefania

Bartolini' \& Alba Ariela Brandes ${ }^{1}$

${ }^{1}$ Department of Medical Oncology, Azienda USL/IRCCS Institute of Neurological Sciences, Bologna, Italy

${ }^{2}$ Department of Neurosciences, University of Messina, Messina, Italy

*Author for correspondence: lidia.gatto83@gmail.com

Glioneuronal tumors are very rare CNS neoplasms that demonstrate neuronal differentiation, composed of mixed glial and neuronal cells. The majority of these lesions are low grade and their correct classification is crucial in order to avoid misidentification as 'ordinary' gliomas and prevent inappropriate aggressive treatment; nevertheless, precise diagnosis is a challenge due to phenotypic overlap across different histologic subtype. Surgery is the standard of therapeutic approach; literature concerning the benefit of adjuvant treatments is inconclusive and a globally accepted treatment of recurrence does not exist. Targetable mutations in the genes $B R A F$ and $F G F R 1 / 2$ are recurrently found in these tumors and could take a promising role in future treatment management.

First draft submitted: 30 January 2020; Accepted for publication: 3 April 2020; Published online:

3 July 2020

Keywords: anaplastic ganglioglioma $\bullet$ central and extraventricular neurocytoma $\bullet$ central neurocytoma $\bullet$ cerebellar liponeurocytoma • desmoplastic infantile ganglioglioma/gangliocytoma • dysembrioplastic neuroepithelial tumor $\bullet$ ganglioglioma $\bullet$ glioneuronal tumors $\bullet$ papillary glioneuronal tumor $\bullet$ paraganglioma $\bullet$ primary diffuse leptomeningeal glioneuronal tumor $\bullet$ rosette-forming glioneuronal tumor

Glioneuronal tumors are a heterogeneous group of CNS neoplasms with neuronal differentiation, exhibiting a pure neuronal pattern or a mixed glial and neuronal phenotype [1]. The revised fourth edition of the World Health Organization (WHO; Geneva, Switzerland) Classification of Tumors of the Central Nervous System, published in 2016, is both a conceptual and practical revolution in brain tumor classification systems, introducing for the first time molecular biology in addition to histology in order to define many tumor entities, according to the era of personalized therapies [2]. According to the $2016 \mathrm{WHO}$ classification, the group of neuronal and mixed glioneuronal neoplasms currently includes dysembryoplastic neuroepithelial tumor (DNET), ganglioglioma, anaplastic ganglioglioma, desmoplastic infantile ganglioglioma/gangliocytoma, central and extraventricular neurocytoma, paraganglioma, cerebellar liponeurocytoma, papillary glioneuronal tumor, rosette-forming glioneuronal tumor and primary diffuse leptomeningeal glioneuronal tumor (DLGNT) [2] (Table 1).

Though this latest version of the WHO classification is recent, in the last few years, many cases have been reported of glioneuronal neoplasms with distinctive morphological features that are still not formally included in any classification $[3,4]$.

Gangliogliomas are the most common histologic subtype, characterized by genetic alterations of the MAP kinase pathway, in particular $B R A F \mathrm{~V} 600 \mathrm{E}$ mutation, other alternative $B R A F$ mutations or fusions, $K R A S$ mutations, NF1 mutations or FGFR mutations or fusions [5]. BRAF V600E mutation represents a valuable diagnostic marker and constitutes a novel and promising therapeutic target for molecularly selected CNS neoplasms in a clinically meaningful way.

Currently the literature of neuroglial tumors is poor and limited to small case series; we thus provide a comprehensive review to summarize presenting symptoms, radiological findings, prognosis and treatment options.

Future Medicine 


\begin{tabular}{|l|l|}
\hline Table 1. Grading of neuronal and mixed neuronal-glial tumors according to the 2016 CNS \\
WHO classification. & Grading \\
\hline Neuronal and mixed neuronal-glial tumors & $\mathrm{I}$ \\
\hline Dysembriplastic neuroepithelial tumor & $\mathrm{I}$ \\
\hline Gangliocytoma & $\mathrm{I}$ \\
\hline Ganglioglioma & $\mathrm{II}$ \\
\hline Anaplastic ganglioglioma & $\mathrm{I}$ \\
\hline Dysplastic cerebellar gangliocytoma & $\mathrm{I}$ \\
\hline Desmoplastic infantile gangliocytoma and ganglioglioma & $\mathrm{I}$ \\
\hline Papillary glioneuronal tumor & $\mathrm{I}$ \\
\hline Rosette-forming glioneuronal tumor & $\mathrm{I}$ \\
\hline Diffuse leptomeningeal glioneuronal tumor & $\mathrm{I}$ \\
\hline Central neurocytoma & $\mathrm{I}$ \\
\hline Extraventricular neurocytoma & $\mathrm{I}$ \\
\hline Cerebellar liponeurocytoma & $\mathrm{I}$ \\
\hline Paraganglioma & \\
\hline
\end{tabular}

\section{Ganglioglioma}

\section{Epidemiology}

Ganglioglioma is a rare well differentiated glioneuronal neoplasm composed of a combination of dysplastic mature ganglion cells with neoplastic glial cells, representing approximately $0.4-1.7 \%$ of all brain tumors [6-10]. It is classified grade I by the 2016 WHO system and generally occurs in children and young adults before age 30 with a slight preponderance in males [11-13].

\section{Clinicopathological \& molecular findings}

Gangliogliomas commonly occur in the supratentorial region, mostly in the temporal lobe (up to $85 \%$ ), followed by the frontal lobe. It can occasionally develop also in the brainstem, cerebellopontine angle, thalamus, optic nerve and spinal cord [14].

Presence of a ganglioglioma in the temporal lobe is particularly epileptogenic; this in part explains the classical clinical presentation with a seizure disorder, generally with a long standing history of epilepsy that is difficult to control medically $[6,13,15,16]$. As reported by Pasquier $e$ t al. in a series of 327 patients with drug-resistant epilepsy, ganglioglioma was found to be the second most common diagnosis [17]. Others presenting symptoms include increased intracranial pressure, headache, nausea, vomiting, personality change, irritability and focal neurological deficit. Cerebellar lesions present with ataxia, headache and hydrocephalus.

Prognosis is favorable and the disease-free survival rate is $97 \%$ at 7.5 years for patients with supratentorial tumors [18] and $88 \%$ for those with spinal cord lesions [19]. However, despite often presenting as low-grade tumors, recurrence or anaplastic progression can occur. Gangliogliomas can develop malignant degeneration of the glial component, thus representing an anaplastic ganglioglioma, a distinct pathological entity corresponding to grade III of WHO classification.

The genetic landscape of ganglioglioma appears to be distinct from several glial and glioneuronal neoplasms and is defined by V600E mutation or alternative BRAF mutations or fusions, RAF1 fusion, KRAS mutation, NF1 mutation or FGFR mutations or fusions.

The activating $B R A F \mathrm{~V} 600 \mathrm{E}$ mutation is common and occurs approximately in $10-60 \%$ of gangliogliomas, with highest prevalence in cortical tumors and lower frequency in spinal cord tumors [20-26]. Conversely, IDH mutation or combined deletion $1 \mathrm{p} / 19 \mathrm{q}$ exclude a diagnosis of ganglioglioma. However, BRAF V600E mutation is not exclusive to ganglioglioma and has been described also in DNET, pilocytic astrocytoma, pediatric $I D H$-wild-type astrocytoma, polymorphous low-grade neuroepithelial tumor of the young, pleomorphic xanthoastrocytoma and epithelioid glioblastoma [26,27].

Several cases of pediatric grade I gangliogliomas have been identified harboring both $H 3 \mathrm{~K} 27 \mathrm{M}$ and $B R A F$ $\mathrm{V} 600 \mathrm{E}$ mutations, characterized by a relatively indolent course compared with diffuse midline glioma $H 3 \mathrm{~K} 27 \mathrm{M}$ mutant [28,29]. While a subgroup of gangliogliomas harbor the well-known BRAF V600E mutation, other genetic alterations are poorly documented. 
Pekmezci et al. performed next-generation sequencing on a cohort of 40 gangliogliomas to provide a comprehensive assessment of the genetic profile of this tumor entity [5]. Thirty-six patients harbored mutations in the MAP kinase pathway; out of these, 18 patients harbored a $B R A F \mathrm{~V} 600 \mathrm{E}$ mutation, five a different $B R A F$ mutation and four had $B R A F$ fusion. In the 13 cases lacking an identifiable $B R A F$ alteration, several genetic mutually exclusive alterations involving KRAS, NF1, FGFR1 and FGFR2 were found. No PRKCA,IDH1,IDH2, TP53, ATRX and $T E R T$ mutations were identified, suggesting that the genetic landscape of ganglioglioma is unique and distinct from the majority of diffuse gliomas, papillary glioneuronal tumors and chordoid gliomas [30,31]. Gangliogliomas sometimes harbor the same BRAF mutations as pilocytic astrocytomas, such as the KIAA1549-BRAF fusion with the appearance of a pilocytic astrocytoma but with foci of gangliocytic differentiation [25].

The prognostic role of BRAF mutation in ganglioglioma is still under investigation.

Dahiya et al. observed that positive BRAF V600E staining is associated with shorter recurrence-free survival [22]. Furthermore, the association between $B R A F$ V600E mutation and $C D K N 2 A$ deletion may be associated with a worse outcome $[32,33]$. CDKN2A is a tumor suppressor gene and its loss acts as a 'secondary hit', which allows malignant behavior, especially when combined with $B R A F$ mutations.

\section{Radiological features}

The neuroimaging appearance is variable, but gangliogliomas often display a mix of solid and cystic components. The computed tomography (CT) imaging of cerebral ganglioglioma is characterized by a nonenhancing cystic lesion, eventually with a ring enhancement or with an enhancing mural nodule. The presence of calcifications is usually extensive and is an important clue in diagnosing of ganglioglioma, especially in the case of small, solid nonenhancing tumors.

On MRI, this tumor usually appears as a solid mass or a mixed solid-cystic mass, iso- to hypointense and hyperintense on T1- and T2-weighted, respectively.

The differential diagnosis includes pleomorphic xantoastrocytoma, pilocytic astrocytoma and hemangioblastoma [34,35]. Although not common, ganglioglioma could be mistaken for a vascular malformation [36].

\section{Treatment options}

The current standard treatment of newly diagnosed gangliogliomas is complete surgical resection [18]. Preoperative and postoperative MRI are useful to define the exact extent of surgery. The tumor location affects the possibility of achieving radical surgery and impacts survival [37-39]. In particular, gangliogliomas of the midline present a poorer outcome, with a higher risk of recurrence and mortality [40]. When gross total resection is achieved and a grade I ganglioglioma is diagnosed, no adjuvant therapies are recommended. If resection is subtotal, a second surgical attempt should be considered, given the prognostic impact of complete resection.

The role of adjuvant postoperative radiation therapy is undefined, it appears to reduce the relapse rate after incomplete resection of high-grade lesions though its benefit is highly debated in cases of total resection or partial removal of low-grade tumors [41-43]. In their retrospective study of 402 patients with ganglioglioma, Rades et al. concluded that gross total resection does not require adjuvant radiation treatment. If resection is subtotal, radiotherapy should be considered because it can improve local control, even if survival advantage at 10 years has not been observed [44]. Radiation therapy can also be considered as an option for 'salvage' treatment in cases of recurrent low-grade tumors [45,46]. Giving the usual young age and long-term survival of individuals with these tumors, before considering the patient for radiotherapy, it is important to weigh its specific long-term toxicity spectrum, in particular neurocognitive sequelae and focal deficits such as optic pathway injury.

The role of chemotherapy is still uncertain; it should be considered for patients with progressive tumors not susceptible to re-resection or reirradiation [47]; nitrosoureas, temozolomide, etoposide, cisplatin, carboplatin and cyclophosphamide have been reported to be effective.

Studies on progressive BRAF V600E-mutated melanomas have shown the effectiveness of the BRAF inhibitors dabrafenib or vemurafenib [48]. Experience with these agents in $B R A F$ V600E-mutated gliomas is limited to case reports [49-55]. Rush et al. described the first case of a brainstem ganglioglioma harboring BRAF V600E mutation that was successfully treated with vemurafenib and vinblastine, achieving complete resolution of symptoms and significant overall decrease in the size of the lesion [49]. Starting from this first experience, other cases of ganglioglioma successfully treated with $R A F$ inhibitors have been accumulated, reinforcing the idea that MAPK pathway inhibitors can be considered as a potential target therapy for these tumors (Table 2). 
Table 2. Positive responses to RAF or MEK/RAF inhibitors in ganglioglioma and anaplastic ganglioglioma.

\begin{tabular}{|c|c|c|c|}
\hline Study (year) & Treatment & Best response & Ref. \\
\hline Rush et al. (2013) & Vemurafenib + vinblastine (ganglioglioma one case) & Partial response & [49] \\
\hline Del Bufalo et al. (2014) & Vemurafenib (ganglioglioma one case) & Partial response & [52] \\
\hline Aguilera et al. (2015) & Vemurafenib (ganglioglioma one case) & $>70 \%$ decrease in tumor size & [50] \\
\hline $\begin{array}{l}\text { Chamberlain et al. } \\
\text { (2016) }\end{array}$ & Dabrafenib (ganglioglioma threecases) & $\begin{array}{l}\text { Stable disease in two patients and a partial response } \\
\text { in one patient }\end{array}$ & [51] \\
\hline Garnier et al. (2019) & Vemurafenib (ganglioglioma one case) & $\begin{array}{l}\text { Partial response manteined } 21 \text { months after } \\
\text { treatment discontinuation }\end{array}$ & [54] \\
\hline Pasqualetti et al. (2019) & Dabrafenib (ganglioglioma one case) & Major response & [55] \\
\hline Lucas Jr. et al. (2014) & Vemurafenib (anaplastic ganglioglioma one case) & Partial response & [56] \\
\hline Melatath et al. (2016) & Dabrafenib (anaplastic ganglioglioma one case) & Complete response & [57] \\
\hline Beland et al. (2018) & Dabrafenib + trametinib (anaplastic ganglioglioma one case) & Complete response & [58] \\
\hline Marks et al. (2018) & Dabrafenib/trametinib (anaplastic ganglioglioma 1 case) & Complete response & {$[60]$} \\
\hline Kaley et al. (2018) & Vemurafenib (anaplastic ganglioglioma one case) & Partial response & [59] \\
\hline Toll et al. (2019) & Dabrafenib/trametinib (anaplastic ganglioglioma one case) & $85 \%$ decrease in tumor size & [61] \\
\hline
\end{tabular}

As expected from experience in other cancers, acquired resistance to $B R A F$ inhibitors may invariably develop, as well as side effects, including skin rash, papillomas and squamous cell carcinomas. To date, there is no valid therapeutic option for patients who progress on vemurafenib or having poor tolerability profile. Studies in $B R A F$ V600E-mutant melanoma showed that a subset of tumors develop resistance to $B R A F$ inhibitors through molecular events reactivating the MAPK pathway, such as NRAS, KRAS or $M E K$ mutations.

This provided rationale for combined $B R A F / M E K$ inhibition, which demonstrated superior outcomes in comparison with $B R A F$ inhibitors monotherapy in BRAF V600E mutant melanoma. Supported by this evidence, several cases of gangliogliomas have been published in which combined treatment with vemurafemib and MEK inhibitor resulted in clinical benefit for patients. Marks et al. reported the case of an anaplastic ganglioglioma with an important skin rash reaction from vemurafenib, who experienced good tolerability and tumor response to the $B R A F / \mathrm{MEK}$ inhibitor combination dabrafenib plus trametinib [62]. Koelsche et al. observed that BRAF V600E-mutated gangliogliomas frequently show lymphocytic infiltrates [63], suggesting an immunogenicity of $B R A F$-mutated gangliogliomas and a potential role for immunotherapy in the future.

Certainly, targeted therapy is very promising in selected subsets of gangliogliomas and further studies are needed to legitimize the use of these novel agents in such a rare group of tumors.

\section{Anaplastic ganglioglioma}

Anaplastic ganglioglioma is a glioneuronal tumor composed of dysplastic ganglion cells and an anaplastic glial component with elevated mitotic activity. It is defined by a WHO grade III component and is associated with significantly worse local control rates, strong potential for distant relapse and short overall survival.

\section{Epidemiology}

The incidence of anaplastic gangliogliomas is very rare and estimated at 0.02 cases/million/year [64], therefore literature is limited to case reports and small retrospective case series.

\section{Clinicopathological \& molecular findings}

Anaplastic ganglioglioma which most often affects children and young adults, is generally unifocal, highly epileptogenic and is known to arise from any part of the CNS, including the spinal cord as well as the cerebral ventricles, even though the temporal lobe is the most common location. Selvanathan et al. reported $27 \%$ of cases arising from temporal lobe, followed by $22 \%$ of cases in the frontal lobe [64]. Unlike gangliogliomas which almost always progress locally, anaplastic gangliogliomas often exhibit diffuse failure within the craniospinal axis and leptomeninges. In particular, in the case series reported by Lucas et al., all three patients experienced leptomeningeal failure [56]. Most commonly the anaplastic transformation occurs in the glial component, resembling a high grade astrocytoma, while 
the neuronal component is relatively benign, although there are reports of anaplastic cells exhibiting both neuronal and astrocytic features as well as sarcomatous differentiation [65].

Anaplastic gangliogliomas rarely occur as de novo tumors (cases not related to previous radiation therapy or prior diagnosis), but more often have been associated with previous subtotal resection or radiotherapy of a low grade ganglioglioma [66-71]. Literature review found only 31 anaplastic gangliogliomas not related to prior radiotherapy or previous diagnosis [72]. The main pathologic findings of anaplastic gangliogliomas include increased mitotic index, pleomorphism, microvascular proliferation, necrosis and gemistocytic differentiation pattern [73]. The $B R A F$ $\mathrm{V} 600 \mathrm{E}$ mutation has been identified in a number of cases of anaplastic ganglioglioma and the incidence of this mutation appears higher in pediatric population.

\section{Radiological features}

Radiological diagnosis is difficult due to the broad spectrum of solid and cystic lesions, the irregularity of contrast enhancement and the variability in the calcification pattern. On MRI, anaplastic gangliogliomas generally appear as a solid mass with a cystic component, hypointense on T1-weighted sequences and hyperintense on T2-weighted images with irregular enhancement after the administration of gadolinium. Proton magnetic resonance spectroscopy $\left({ }^{1} \mathrm{H}-\mathrm{MRS}\right)$, performed to measure the levels of metabolites in the tumors, can reveal a high choline peak relative to a high $\mathrm{N}$-acetylaspartate/creatine ration and increased lactate and lipid levels, which suggest anaplastic behavior [13].

\section{Treatment options}

Gross total resection is considered the standard of care for anaplastic gangliogliomas [64,74,75]. However, even after a complete surgical resection tumor recurrence can occur; thus, many centers recommend adjuvant radiotherapy or chemo-radiotherapy to improve tumor control and survival [64,74,75]. Even after adjuvant treatment survival is poor.

In a retrospective study from the the National Cancer Institute's Surveillance, Epidemiology and End Results database, Selvanathan et al. analyzed a cohort of 58 adult and pediatric anaplastic gangliogliomas [64]. The median overall survival was 28.5 months and univariate and multivariate analysis identified surgical resection and unifocal disease as prognostic factors that could impact survival. No statistically significant benefit was found in overall survival, but only a trend toward longer survival has been observed in patients who received adjuvant radiotherapy. As admitted by the authors themselves, however, in this study, the small sample size may have influenced statistical power and the information on tumor size, type of surgical resection and the use of radiotherapy or chemotherapy is limited.

Another large series published is the French Brain Tumor Database study, which included 43 cases of anaplastic ganglioglioma [57]. In this series, the total resection was achieved in $58.8 \%$ of patients. Adjuvant radiotherapy with concomitant temozolomide - the standard Stupp protocol of combined chemo-radiotherapy for glioblastoma - was performed in approximately half of the patients. Adjuvant radiotherapy alone was administered in approximately $30 \%$ of patients and adjuvant chemotherapy alone without radiotherapy in $6 \%$ of patients. Tumor recurrence rate at 5 years was about $100 \%$ and median overall survival was 24.7 months. The subgroup with the best overall survival (37.3 months) was that of patients treated with gross total resection and adjuvant radio-chemotherapy [74].

Mallick et al. [75] performed a search of PubMed to find all the publications related to anaplastic ganglioglioma to establish the optimum treatment of this tumor type. A total of 40 publications with overall 69 patients were found eligible. It has been observed that patients undergoing a gross total resection have a significantly better overall survival compared with those with a subtotal resection, but neither adjuvant radiation, nor chemotherapy were found to have any impact on progression-free or overall survival. Also, this analysis failed to elicit any advantage of adjuvant radiation and chemotherapy and the authors concluded that the small sample and the heterogeneity of treatments may have affected the results. Therefore, they suggest, as reasonable approach, radiation for patients who received a gross total resection and adjuvant radiation or chemotherapy or a combination of both in case of subtotal resection or for disease at eloquent location. It is important to note that in all these large case series, median overall survival is poor and does not exceed 30 months.

Positive responses to targeted therapy with $R A F$ inhibitor or MEK/RAF inhibitor combination therapy have been reported in anaplastic ganglioglioma refractory to other treatments (Table 2) [56,58,60,61]. Kaley et al. published the Phase II, histology-independent VE-BASKET trial for BRAF V600-mutant nonmelanoma patients. Patients with $B R A F$ V600-mutant glioma received vemurafenib $960 \mathrm{mg}$ twice per day until they experienced disease progression, unacceptable adverse effects or withdrew. Twenty-four patients with glioma, including malignant diffuse glioma 
( $\mathrm{n}=11$; six glioblastoma and five anaplastic astrocytoma), pleomorphic xanthoastrocytoma $(\mathrm{n}=7)$, anaplastic ganglioglioma $(\mathrm{n}=3)$, pilocytic astrocytoma $(\mathrm{n}=2)$ and high-grade glioma, not otherwise specified $(\mathrm{n}=1)$, were treated. Objective response rate was $25 \%$ and the median progression-free survival was 5.5 months. In particular, one patient diagnosed with anaplastic ganglioglioma obtained a partial response and was treated for 13.8 months for a confirmed clinical benefit rate of $33 \%$ (95\% CI: 4.3-77.7\%) [59]. On the basis of this evidence, a larger prospective study is needed.

\section{Dysembryoplastic neuroepithelial tumor}

A DNET is a grade I mixed neuronal-glial tumor, causing drug-resistant epilepsy that occurs in the cerebral cortex of children and young adults with a predilection for the temporal lobe.

\section{Epidemiology}

The incidence of DNETs is 0,03 person-year per 100.000 , with a peak in the range between 10 and 14 years.

\section{Clinicopathological \& molecular findings}

It is characterized by multinodular architecture consisting of columns of oligodendroglial cells interspersed with a mucoid matrix with floating neurons [76,77]. Histological variants of DNET have been described, with additional glial cell component and a nodular appearance [78-81]; the simplest form, instead, consists of unique glioneuronal elements. DNET cells are positive for S100 protein, synaptofisin, neuronal nuclei, neurite outgrowth inhibitor OLIG2 and MAP 2, but negative for GFAP.

DNETs are stable or very slow growing and require no postoperative adjuvant therapy [82]. Long-term clinical follow-up usually demonstrates an extremely low rate of recurrence; however, the rare cases of recurrences and malignant transformations legitimize the need for MRI surveillance, mostly after incomplete resection.

DNETs share with gangliogliomas FGFR1 and BRAF V600E mutations, the latter found in $27-51 \%$ of cases (3283). The prevalence of $B R A F \mathrm{~V} 600 \mathrm{E}$ mutations is higher in the complex type and in the extratemporal location (i.e., tumors in midbrain or brainstem). Prabowo et al. observed that the presence of BRAF V600E mutation is significantly associated with an mTOR pathway overactivation in ganglioglioma and DNET [32]. In ganglioglioma and DNETs, the presence of BRAF V600E mutation has been associated with the expression of phosphorylated ribosomal S6 protein (pS6), a marker of overactivation of the mTOR pathway and a key regulator of cell growth and proliferation. Interestingly, on the basis of this association, mTOR targeted treatment may be developed.

\section{Radiological features}

Cortical topography and the absence of edema and mass effect are the most important criteria for differentiating DNETs from diffuse gliomas. In conventional MRI, DNETs present as multiple or single cystic lesions, hypointense on T1-weighted and hyperintense on both T2-weighted and fluid attenuated inversion-recovery MRI. Noncystic tissue is hypointense on T1-weighted images and hyperintense on T2-weighted and fluid attenuated inversionrecovery images. On both CT and MRI, in approximately $20 \%$ to a third of the patients, a nodular, ring-like or heterogeneous contrast enhancement may be observed. The enhancement can also be observed during the followup in a previously nonenhancing tumor, these variations being usually considered ischemic and/or hemorrhagic changes rather than an expression of malignancy.

Fiber tractography is also important for the differential diagnosis versus low-grade glioma. In DNETs, a pattern of displacement of the fiber tracts is observed, while gliomas spread along white matter tracts.

Advanced MRI techniques - in other words, diffusion, perfusion and spectroscopy - may be useful in the differential diagnosis for DNET versus other low-grade gliomas. Unlike low-grade gliomas, on diffusion-weighted MRI, DNETs generally present a high apparent diffusion coefficient as the expression of low cellular density; such values range between 2.38 and 2.78. On perfusion-MRI, DNETs have a lower relative cerebral blood volume value than diffuse gliomas (range 0.66-0-99), due to the presence of 'floating neurons' and high-water content. Proton magnetic resonance spectroscopy profile is characterized by a not decreased $\mathrm{N}$-acetyl-aspartate/choline (NAA/Cho) ratio, when compared with diffuse gliomas and a high myoinositol/creatine ratio, which ranges between 0.19 and 0.57. Identification of FGFR1 and BRAF V600E mutations limits the risk of misdiagnosis [83,84].

\section{Treatment options}

Complete tumor resection leads to a long-term seizure control [85-87], with 70-90\% of resected patient seizure free. 


\section{Central neurocytoma \& extraventricular neurocytoma}

\section{Epidemiology}

Central neurocytomas $(\mathrm{CNs})$ represent about $0.1-0.5 \%$ of all brain tumors. CNs are more frequent among young adults, with an incidence peak at about 30 years [88-93]. In several studies, it has been observed that there is a higher incidence of $\mathrm{CNs}$ in Korea, Japan and India, which may be attributable to genetic interracial differences [94-97].

\section{Clinicopathological \& molecular findings}

$\mathrm{CN}$ is composed of uniform round cells with neuronal differentiation and low proliferation index and it corresponds histologically to grade II. It is usually supratentorial, occurring in the lateral ventricles or in the third ventricle, especially in the region of the foramen of Monro. Attachment to the septum pellucidum seems to be a feature of the tumor. CN may manifest as obstructive hydrocephalus with signs of increased intracranial pressure or with distinct focal deficit.

The MIB-1 Labeling Index (MIB-1 LI) is an important prognostic tool for $\mathrm{CN}$ and is an accurate indicator of tumor relapse and tumor grade. Imber $e t$ al. analyzed progression-free survival and overall survival in a cohort of 28 patients and found that low MIB-1 LI $(<4 \%)$ correlates with longer progression-free survival and overall survival [98]. Similarly, Chen $e t$ al. found that a MIB-1 LI $>2 \%$ may indicate a more aggressive disease course [99].

Many types of genetic mutations have been associated with $\mathrm{CNs}$, in particular overexpression of $N-M Y C, I G F 2$, PTEN, PDGF-D and NRG-2 [100].

\section{Radiological features}

The radiological features of $\mathrm{CN}$ are nonspecific; $\mathrm{CT}$ scans usually demonstrate a hyperdense mass in the lateral ventricles. MRI shows a peri-ventricular mass, hypointense or isointense on T1-weighed images and isointense or hyperintense on T2-weighed. Contrast enhancement can be variable, generally moderate-to-strong [91,101-103].

\section{Treatment options}

The gold standard of treatment is gross total resection, which often allows for a very high rate of tumor control and long-term survival. In case of subtotal resection and/or an elevated MIB-1 LI, there may be an indication for adjuvant radiotherapy. The use of chemotherapy is debated; the literature is limited to case reports and is very heterogeneous with regard to scheme of chemotherapy and timing of initiation - upfront versus salvage treatment. Brandes et al. reported three cases of neurocytoma treated with chemotherapy. Disease progressed in two patients after surgery and adjuvant radiotherapy and in one patient after surgery. The treatment regimen included etoposide, cisplatin and cyclophosphamide. Disease stabilization was observed in two patients and complete response occured in one patient with long maintenance of the response [104]. Johnson et al. reported the case of a young woman successfully treated with temozolomide. The patient had a recurrence 6 years after initial treatment with gross-total resection, this recurrence was treated with repeat surgery followed by temozolomide and concurrent radiation for 5 weeks. Fourteen years after the first diagnosis and 6 years 9 months after the recurrence, the patient was clinically stable [105].

Imber $e t$ al. analyzed a cohort of 28 patients treated at their institution between 1995 and 2014. In their case series, four patients with recurrent $\mathrm{CN}$ received salvage chemotherapy. One patient, treated with CCNU, had significant radiographic response with subsequent disease stabilization. Two patients, treated with temozolomide, experienced 3 years of tumor stabilization followed by tumor progression in one case and slow progressive tumor despite chemotherapy in the other case, respectively [98].

Occasionally, neurocytoma occurs outside the ventricles and is called extraventricular neurocytoma, an atypical form that arises in the spinal cord or cerebellum. It is associated with worse outcome due to a higher proliferative index and recur within a relatively short period of follow-up [106-109].

\section{Diffuse leptomeningeal glioneuronal tumor}

DLGNT is a new entity which has been included in the 2016 update of the WHO classification, characterized by predominant and widespread leptomeningeal growth and an oligodendroglial-like cytology with elements of neuronal differentiation. It has been described a high rate of $K I A A 1549-B R A F$ gene fusion or $1 \mathrm{p} / 19 \mathrm{q}$ co-deletion in the absence of $I D H$ mutation. Due to the limited cases in literature, the WHO classification has not been assigned, to date, a grade to this tumor entity. 


\section{Clinicopathological \& molecular findings}

DLGNT was first described in 2010 [110], prior to that it was reported as 'disseminated oligodendroglial-like leptomeningeal tumor of childhood' [111]. The cellular origin is unclear; the absence of brain parenchymal lesions suggests an origin from neuroepithelial cells scattered in the meninges. The wide spectrum of histological and radiological features can make this tumor entity difficult to diagnose. This tumor is characterized by diffuse leptomeningeal growth, often without a recognizable parenchymal lesion (commonly in the spinal cord), with an incidence peak in children and young adults. Histology demonstrates a monomorphic clear cell glial morphology, reminiscent of oligodendroglioma, with immunostaining positivity for GFAP, OLIG2, S100 and synaptophysin and negativity for IDH1. Microscopic cerebral fluid examination demonstrates elevated protein levels although cytology is often negative.

A newly recognized entity is the multinodular and vacuolated pattern, a low-grade purely neuronal tumor affecting adults, situated in the cerebral hemispheres, most often in the temporal lobe, composed of tumor cells exhibiting nuclear immunolabeling for the $\mathrm{HuC} / \mathrm{HuD}$ neuronal antigens and expression of other neuronal markers, in particular synaptophysin and neurofilament while chromogranin is variable [112]. This tumor commonly harbors $B R A F$ fusions as well as $1 \mathrm{p} / 19 \mathrm{q}$ co-deletion, $1 \mathrm{p}$ or $19 \mathrm{q}$ solo deletion [113]. IDH mutation is absent.

The prognosis is variable, with a majority of low-grade tumors showing relatively slow progression and a subset of tumors with a more aggressive course, that shows features of anaplasia. Studies have indicated that $1 \mathrm{p} / 19 \mathrm{q}$ co-deletion or $1 \mathrm{p} / 19 \mathrm{q}$ solo deletion demonstrate a more aggressive biological behavior and are sensitive to chemotherapy, especially temozolomide [114].

\section{Radiological features}

The MRI findings show two distinct patterns: one of diffuse leptomeningeal enhancing, the second of 'small cysts' implants scattered all over the brain and spinal cord, especially along the surface of the posterior fossa and basal brain regions, giving the distinctive neuroimaging profile of a diffuse 'microcystic meningoencephalopathy'.

\section{Treatment options}

Complete surgical resection is difficult; radiotherapy and chemotherapy are valid option most notably in more aggressive DLGNTs with high ki67 rate. Chemotherapy regimens include carboplatin and vincristine, temozolomide, etoposide and bevacizumab [114].

\section{Rosette-forming glioneuronal tumor}

Rosette forming glioneuronal tumor is a grade I neoplasm that has biphasic cytoarchitecture with two elements: neurocytes forming rosettes/perivascular psuedorosettes and astrocytic cells resembling pilocytic astrocytoma [115117].

\section{Epidemiology}

The incidence rate is not yet available, with about 100 cases reported in literature.

\section{Clinicopathological \& molecular findings}

The term rosette-forming glioneuronal tumor was used for the first time by Komori et al. in 1998 [118]; it most commonly occurs in young adults, occupying the fourth ventricle. Until the description of two cases in the optic chiasm [119] and spinal cord [120], respectively, these tumors were believed to originate only in the posterior fossa. To date, rare locations in the pineal region, cerebellar vermis, pons and septum pellucidum have also been observed [121,122].

The spectrum of clinical symptoms is wide, including headaches, visual disturbances, nausea and vomiting, vertigo, ataxia, cervical pain and neck rigidity [123].

They are low-grade tumors with lack of atypia and low Ki67 labeling indices [124]. Molecular studies of rosetteforming glioneuronal tumors are few and have revealed only two recurrent genetic alterations; PIK3CA or FGFRI mutations. IDH1/2 mutation and $1 \mathrm{p} / 19 \mathrm{q}$ co-deletion are absent. In particular, PIK3CA mutations are missense mutations in exon 20 (nucleotide $3140 \mathrm{~A}[\mathrm{G}, \mathrm{H} 1047 \mathrm{R}$ ) and in exon 9 (nucleotide $1624 \mathrm{~A}[\mathrm{G}, \mathrm{E} 542 \mathrm{~K}$ ), while FGFR1 mutations have been found in two patients: the FGFR1 N546K mutation (AAC->AAG, Asn->Lys) was found in a 27-year-old woman and the K656E (AAG->GAG, Lys-> Glu) in a 12-year-old boy. The presence 
of FGFR1 mutations in rosette-forming glioneuronal tumors may suggest a molecular similarity with pilocytic astrocytoma $[125,126]$.

\section{Radiological features}

These tumors are relatively circumscribed, with calcifications and a ring-shaped contrast enhancement, hypointense on T1 and hyperintense or isointense on T2-weighted MRI sequences [123].

\section{Treatment options}

Management is usually through surgery with gross total resection providing better prognosis. Nevertheless, tumor location and its frequent extension within the adjacent structures does not always permit a total resection without surgical morbidity or neurological dysfunction. Due to their indolent nature, a subtotal removal is also considered acceptable, whereas an aggressive approach can increase morbidity. In this tumor type, the recurrence rate is extremely low, with only four cases of recurrence reported as of now [124,125,127].

\section{Conclusion \& future perspective}

Glioneuronal tumors represent a heterogeneous group of neoplasms that exhibit neuronal differentiation, with a pure neuronal differentiation pattern or with a mixed glial and neuronal phenotype [1]. The numerous new entities recently described in the literature suggests that the wide spectrum of neuronal and glioneuronal neoplasms is far from being exhaustively documented. Given the rarity of these neoplasms, the benefit of various management

\section{Executive summary}

\section{Epidemiology \& pathology of neuronal \& glioneuronal tumors}

- Neuronal and glioneuronal tumors are a heterogeneous group of CNS neoplasms that demonstrate neuronal differentiation, with either a pure neuronal or a mixed glial and neuronal phenotype.

- Compared with other brain tumors they are very rare and therefore not well characterized; they mainly occur in children and young adults, predominantly have a low-grade histology, with an indolent course and long-term survival after surgical resection.

- Onset symptoms depend on tumor location, the most common being pharmacoresistant seizures, followed by intracranial hypertension and focal deficits.

- Within this group, ganglioglioma is the most common histologic subtype and consequently also the best studied, whose molecular biology is well known, which paves the way for new target therapies.

- The excellent prognosis and the malignant transformation potential of the glial component are the two most remarkable findings in ganglioglioma.

- Radiological diagnosis presents a challenge due to the overlap of imaging features across different histotypes, which complicates diagnosis.

- The MRI appearance of glioneuronal tumors could be very variable across the diverse histotypes but these tumors, expecially WHO grade I, most often demonstrate similar neuroradiological findings: a solid-cystic mass with enhanced peripheral ring and diffuse pattern of calcification.

\section{Treatment options}

- The conventional treatment for low-grade tumors involves surgical resection.

- Adjuvant treatments, though their exact role is unknown, may be considered individually based on pathological subtypes and a proper assessment of risks and benefits. Adjuvant radiotherapy, in particular, may be recommended in case of incomplete surgical resection and high proliferative index.

- The role of chemotherapy is unclear and generally reserved for savage therapy, with a vast heterogeneity of schemes across the diverse histotypes but also within the same type of tumor.

- In case of disease progression, despite aggressive treatment with radiation and chemotherapy, BRAF and MEK inhibitors represent a promising therapeutic option that may improve the disease course of glioneuronal tumors in a clinically meaningful way.

\section{Future perspective $\&$ conclusion}

- Due to the rarity of these neoplasms, available studies comparing the benefit of various management strategies are few. Thus, the level of evidence of recommendations is low.

- Identification of the molecular basis of this niche diseases is a challenge; prospective clinical studies with BRAF-MEK inhibitors should be conducted, not only in patients who have already failed the radiotherapy and chemotherapy, but also in the adjuvant setting where traditional therapies are associated with acute and long-term toxicities, shifting the therapeutic algorithm toward an earlier integration of molecularly targeted agents. 
strategies is not clearly established and the level of evidence of recommended treatments is low. Currently the interest in this class of brain tumors is high, thanks to the increasing knowledge of molecular biology and the remarkable responses to targeted therapies observed, especially in those with $B R A F$-mutated tumors.

Financial \& competing interests disclosure

The authors have no relevant affiliations or financial involvement with any organization or entity with a financial interest in or financial conflict with the subject matter or materials discussed in the manuscript apart from those disclosed.

No writing assistance was utilized in the production of this manuscript.

\section{Open access}

This work is licensed under the Attribution-NonCommercial-NoDerivatives 4.0 Unported License. To view a copy of this license, visit http://creativecommons.org/licenses/by-nc-nd/4.0/

\section{References}

Papers of special note have been highlighted as: $\bullet \bullet$ of considerable interest

1. Brat DJ, Perry A. Neuronal and glioneuronal neoplasms. Practical Surgical Neuropathology: A Diagnostic Approach (2nd Edition). Elsevier, Amsterdam, The Netherlands, 183-217 (2008).

2. Louis DN, Perry A, Reifenberger G et al. The 2016 World Health Organization classification of tumors of the central nervous system: a summary. Acta Neuropathol. 131(6), 803-820 (2016).

•• The last WHO Classification of the CNS tumors.

3. Lucas CG, Villanueva-Meyer JE, Whipple N et al. Myxoid glioneuronal tumor, PDGFRA p.K385-mutant: clinical, radiologic, and histopathologic features. Brain Pathol. 30(3), 479-494 (2019)

4. Yamada S, Nobusawa S, Yamazaki T et al. An epilepsy-associated glioneuronal tumor with mixed morphology harboring FGFRI mutation. Pathol. Int. 69(6), 372-377 (2019).

5. Pekmezci M, Villanueva-Meyer JE, Goode B et al. The genetic landscape of ganglioglioma. Acta Neuropathol. Commun. 6(1), 47 (2018).

-• Accurate analysis of detectable molecular alterations in gangliogliomas.

6. Tomita T, Volk JM, Shen W, Pundy T. Glioneuronal tumors of cerebral hemisphere in children: correlation of surgical resection with seizure outcomes and tumor recurrences. Childs Nerv. Syst. 32(10), 1839-1848 (2016).

7. Luyken C, Blümcke I, Fimmers R, Urbach H, Wiestler OD, Schramm J. Supratentorial gangliogliomas: histopathologic grading and tumor recurrence in 184 patients with a median follow-up of 8 years. Cancer 101(1), 146-155 (2004).

8. Kalyan-Raman UP, Olivero WC. Ganglioglioma: a correlative clinico- pathological and radiological study of ten surgically treated cases with follow-up. Neurosurgery 20(3), 428-433 (1987).

9. Pandita A, Balasubramaniam A, Perrin R, Shannon P, Guha A. Malignant and benign ganglioglioma: a pathological and molecular study. Neuro-oncol. 9(2), 124-134 (2007).

10. Demierre B, Stichnoth FA, Hori A, Spoerri O. Intracerebral ganglioglioma. J. Neurosurg. 65(2), 177-182 (1986).

11. Blümcke I, Wiestler OD. Gangliogliomas: an intriguing tumor entity associated with focal epilepsies. J. Neuropathol. Exp. Neurol. 61(7), 575-584 (2002).

12. Ulutin HC, Ongürü O, Pak Y. Postoperative radiotherapy for gangli- oglioma; report of three cases and review of the literature. Minim. Invasive Neurosurg. 45(4), 224-227 (2002).

13. Im SH, Chung CK, Cho BK et al. Intracranial ganglioglioma: preoperative characteristics and oncologic outcome after surgery. $J$. Neurooncol. 59(2), 173-183 (2002).

14. Janjua MB, Ivasyk I, Pisapia DJ, Souweidane MM. Ganglioglioma of brain stem and cervicomedullary junction: a 50 years review of literature. J. Clin. Neurosci. 44, 34-46 (2017).

15. Walker DA, Liu J, Kieran M et al. A multi-disciplinary consensus statement concerning surgical approaches to low-grade, high-grade astrocytomas and diffuse intrinsic pontine gliomas in childhood (CPN Paris 2011) using the Delphi method. Neuro Oncol. 15(4), 462-468 (2013).

16. Tomasello C, Franceschi E, Tosoni A, Brandes A. Gangliogliomas: recent advances in classification and treatment. Future Neurol. 5(4), 557-568 (2010).

17. Pasquier B, Péoc HM, Fabre-Bocquentin B et al. Surgical pathology of drug-resistant partial epilepsy. A 10-year-experience with a series of 327 consecutive resections. Epileptic Disord. 4(2), 99-119 (2002).

18. Luyken C, Blumcke I, Fimmers R, Urbach H, Wiestler OD, Schramm J. Supratentorial gangliogliomas: histopathologic grading and tumor recurrence in 184 patients with a median follow-up of 8 years. Cancer 101(1), 146-155 (2004).

19. Jallo GI, Freed D, Epstein FJ. Spinal cord gangliogliomas: a review of 56 patients. J. Neurooncol. 68(1), 1-77 (2004). 
20. Chappe C, Padovani L, Scavarda D et al. Dysembryoplastic neuroepithelial tumors share with pleomorphic xanthoastrocytomas and gangliogliomas BRAF(V600E) mutation and expression. Brain Pathol. 23(5), 574-583 (2013).

21. Chen $\mathrm{X}$, Pan $\mathrm{C}$, Zhang $\mathrm{P}$ et al. BRAF V600E mutation is a significant prognosticator of the tumour regrowth rate in brainstem gangliogliomas. J. Clin. Neurosci. 46, 50-57 (2017).

22. Dahiya S, Haydon DH, Alvarado D, Gurnett CA, Gutmann DH, Leonard JR. BRAF(V600E) mutation is a negative prognosticator in pediatricganglioglioma. Acta Neuropathol. 125(6), 901-910 (2013).

23. Donson AM, Kleinschmidt-DeMasters BK, Aisner DL et al. Pediatric brainstem gangliogliomas show BRAF(V600E) mutation in a high percentage of cases. Brain Pathol. 24(2), 173-183 (2014).

24. Gessi M, Dörner E, Dreschmann V et al. Intramedullary gangliogliomas: histopathologic and molecular features of 25 cases. Hum. Pathol. 49, 107-113 (2016).

25. Gupta K, Orisme W, Harreld JH et al. Posterior fossa and spinal gangliogliomas form two distinct clinicopathologic and molecular subgroups. Acta Neuropathol. Commun. 2, 18 (2014).

26. Schindler G, Capper D, Meyer J et al. Analysis of BRAF V600E mutation in 1,320 nervous system tumors reveals high mutation frequencies in pleomorphic xanthoastrocytoma, ganglioglioma and extra-cerebellar pilocytic astrocytoma. Acta Neuropathol. 121(3), 397-405 (2011).

27. Zhang J, Wu G, Miller CP et al. Whole-genome sequencing identifies genetic alterations in pediatric low-grade gliomas. Nat. Genet. 45(6), 602-612 (2013).

28. Pages M, Beccaria K, Boddaert $\mathrm{N}$ et al. Co-occurrence of histone $H 3 \mathrm{~K} 27 \mathrm{M}$ and $B R A F \mathrm{~V} 600 \mathrm{E}$ mutations in paediatric midline grade I ganglioglioma. Brain Pathol. 28(1), 103-111 (2018).

29. Louis DN, Giannini C, Capper D et al. cIMPACT-NOW update 2: diagnostic clarifications for diffuse midline glioma, $H 3$ K27M-Mutant and diffuse astrocytoma/anaplastic astrocytoma, IDH-mutant. Acta Neuropathol. 135(4), 639-642 (2018).

30. Bridge JA, Liu XQ, Sumegi J et al. Identification of a novel, recurrent SLC44A1-PRKCA fusion in papillary glioneuronal tumor. Brain Pathol. 23(2), 121-128 (2013).

31. Cancer Genome Atlas Research Network, Brat DJ, Verhaak RG et al. Comprehensive, integrative genomic analysis of diffuse lower-grade gliomas. N. Engl. J. Med. 372(26), 2481-2498 (2015).

32. Prabowo AS, Iyer AM, Veersema TJ et al. BRAF V600E mutation is associated with mTOR signaling activation in glioneuronal tumors. Brain Pathol. 24(1), 52-66 (2014).

33. Lassaletta A, Zapotocky M, Mistry M et al. Therapeutic and prognostic implications of BRAF V600E in pediatric low-grade gliomas. J. Clin. Oncol. 35(25), 2934-2941 (2017).

34. Zentner J, Wolf HK, Ostertun B et al. Gangliogliomas: clinical, radiological and histopathological findings in 51 patients. J. Neurol. Neurosurg. Psychiatry 57(12), 1497-1502 (1994).

35. Demir MK, Yapicier O, Yilmaz B, Kiliç T. Magnetic resonance imaging findings of mixed neuronal-glial tumors with pathologic correlation: a review. Acta Neurol. Belg. 118(3), 379-386 (2018).

36. Baltuch GH, Farmer JP, Meagher-Villemure K, Montes JL, O'Gorman AM. Ganglioglioma presenting as a vascular lesion in a 10-year old boy. Case report. J. Neurosurg. 79(6), 920-923 (1993).

37. Miller DC, Lang FF, Epstein FJ. Central nervous system gangliogliomas. Part 1: pathology. J. Neurosurg. 79(6), 859-866 (1993).

38. Matsumoto K, Tamiya T, Ono T, Furuta S, Asari S, Ohmoto T. Cerebral gangliogliomas: clinical characteristics, CT and MRI. Acta Neurochir. 141(2), 135-141 (1999).

39. Stokland T, Liu JF, Ironside JW et al. A multivariate analysis of factors determining tumor progression in childhood low-grade glioma: a population-based cohort study (CCLG CNS9702). Neuro Oncol. 12(12), 1257-1268 (2010).

40. Terashima K, Chow K, Jones J et al. Long-term outcome of centrally located low-grade glioma in children. Cancer 119(14), 2630-2638 (2013).

41. Selch MT, Goy BW, Lee SP et al. Gangliogliomas: experience with 34 patients and review of the literature. Am. J. Clin. Oncol. 21(6), 557-564 (1998).

42. Packer RJ. Radiation therapy for pediatric low-grade gliomas: survival and sequelae. Curr. Neurol. Neurosci. Rep. 10(1), 10-13 (2010).

43. Merchant TE, Kun LE, Wu S, Xiong X, Sanford RA, Boop FA. Phase II trial of conformal radiation therapy for pediatric low-grade gliomas. J. Clin. Oncol. 27(22), 3598-3604 (2009).

44. Rades D, Zwick L, Leppert J et al. The role of postoperative radiotherapy for the treatment of gangliogliomas. Cancer 116(2), 432-442 (2010).

45. Soffietti R, Rudà R, Reardon D. Rare glial tumors. In: Handbook of Clinical Neurology, Vol 134 (3rd Series). Berger MS, Weller M (Eds). Elsevier, Amsterdam, The Netherlands, 399-415 (2016).

46. Liauw SL, Byer JE, Yachnis AT, Amdur RJ, Mendenhall WM. Radiotherapy after subtotally resected or recurrent ganglioglioma. Int. J. Radiat. Oncol. Biol. Phys. 67(1), 244-247 (2007). 
47. Mohile N, Raizer JJ. Chemotherapy for glioneuronal tumors. In: Handbook of Brain Tumor Chemotherapy. Newton HB (Ed.). Elsevier, Amsterdam, The Netherlands, 432-438 (2006).

48. Chapman PB, Hauschild A, Robert C et al. Improved survival with vemurafenib in melanoma with BRAF V600E mutation. N. Engl. J. Med. 364(26), 2507-2516 (2011).

49. Rush S, Foreman N, Liu A. Brainstem ganglioglioma successfully treated with vemurafenib. J. Clin. Oncol. 31(10), e159-e160 (2013).

-• First case of $B R A F$-mutated ganglioglioma successfully treated with vemurafenib.

50. Aguilera D, Janss A, Mazewski C et al. Successful retreatment of a child with a refractory brainstem ganglioglioma with vemurafenib. Pediatr. Blood Cancer 63(3), 541-543 (2015).

51. Chamberlain MC. Recurrent ganglioglioma in adults treated with BRAF inhibitors. CNS Oncol. 5(1), 27-29 (2016).

52. Del Bufalo F, Carai A, Figà-Talamanca L et al. Response of recurrent BRAF V600E mutated ganglioglioma to Vemurafenib as single agent. J. Transl. Med. 12, 356 (2014).

53. Shih KC, Shastry M, Williams JT et al. Successful treatment with dabrafenib (GSK2118436) in a patient with ganglioglioma. J. Clin. Oncol. 32(29), e98-e100 (2014).

54. Garnier L, Ducray F, Verlut C et al. Prolonged response induced by single agent vemurafenib in a BRAF V600E spinal ganglioglioma: a case report and review of the literature. Front. Oncol. 9, 177 (2019).

55. Pasqualetti F, Gonnelli A, Molinari A et al. Treatment with dabrafenib in a patient with BRAF mutated recurrent ganglioglioma. Neuro Oncol. 20(Suppl. 3), iii258 (2018).

56. Lucas JT Jr, Huang AJ, Mott RT, Lesser GJ, Tatter SB, Chan MD. Anaplastic ganglioglioma: a report of three cases and review of literature. J. Neuro Oncol. 123(1), 171-177 (2015).

57. Matsuzaki K, Uno M, Kageji T, Hirose T, Nagahiro S. Anaplastic ganglioglioma of the cerebellopontine angle. Case report. Neurol. Med. Chir. 45(11), 591-595 (2005).

58. Beland B, Tsang RY, Sutherland G. Unprecedented response to combination BRAF and MEK inhibitors in adult anaplastic ganglioglioma. J. Neurooncol. 137(3), 667-669 (2018).

59. Kaley $\mathrm{T}$, Touat $\mathrm{M}$, Subbiah V et al. BRAF Inhibition in BRAF ${ }^{\mathrm{V} 600}$-Mutant Gliomas: results from the VE-BASKET Study. J. Clin. Oncol. 36(35), 3477-3484 (2018).

60. Marks AM, Bindra RS, DiLuna ML et al. Response to the BRAF/MEK inhibitors dabrafenib/trametinib in an adolescent with a $B R A F$ V600E mutated anaplastic ganglioglioma intolerant to vemurafenib. Pediatr. Blood Cancer 65(5), e26969 (2018).

61. Toll SA, Tran HN, Cotter J et al. Sustained response of three pediatric BRAF(V600E) mutated high-grade gliomas to combined BRAF and MEK inhibitor therapy. Oncotarget 10(4), 551-557 (2019).

62. Marks AM, Bindra RS, DiLuna ML et al. Response to the $B R A F /$ MEK inhibitors dabrafenib/trametinib in an adolescent with a $B R A F$ V600E mutated anaplastic ganglioglioma intolerant to vemurafenib. Pediatr. Blood Cancer 65(5), e26969 (2018).

63. Koelsche C, Wohrer A, Jeibmann A et al. Mutant BRAF V600E protein in ganglioglioma is predominantly expressed by neuronal tumor cells. Acta Neuropathol. 125(6), 891-900 (2013).

64. Selvanathan SK, Hammouche S, Salminen HJ, Jenkinson MD. Outcome and prognostic features in anaplastic ganglioglioma: analysis of cases from the SEER database. J. Neurooncol. 105(3), 539-545 (2011).

-• Largest case series of anaplastic ganglioglioma.

65. Sasaki A, Hirato J, Nakazato Y, Tamura M, Kadowaki H. Recurrent anaplastic ganglioglioma: pathological characterization of tumor cells. Case report. J. Neurosurg. 84(6), 1055-1059 (1996).

66. Mittler MA, Walters BC, Fried AH, Sotomayor EA, Stopa EG. Malignant glial tumor arising from the site of a previous hamartoma/ganglioglioma: coincidence or malignant transformation? Pediatr. Neurosurg. 30(3), 132-134 (1999).

67. Kurian NI, Nair S, Radhakrishnan VV. Anaplastic ganglioglioma: case report and review of the literature. Br. J. Neurosurg. 12(3), 277-280 (1998).

68. Campos MG, Zentner J, Ostertun B, Wolf HK, Schramm J. Anaplastic ganglioglioma: case report and review of the literature. Neurol. Res. 16(4), 317-320 (1994)

69. Whittle IR, Mitchener A, Atkinson HD, Wharton SB. Anaplastic progression in low grade glioneural neoplasms. Acta Neuropathol. 104(2), 215 (2002).

70. Danjoux M, Sabatier J, Uro-Coste E, Roche H, Delisle MB. Anaplastic temporal ganglioglioma with spinal metastasis. Ann. Pathol. 21(1), 55-58 (2001).

71. Karremann M, Pietsch T, Janssen G, Kramm CM, Wolff JE. Anaplastic gaglioglioma in children. J. Neuro Oncol. 92(2), 157-163 (2009).

72. DeMarchi R, Abu-Abed S, Munoz D, Loch Macdonald R. Malignant ganglioglioma: case report and review of literature. J. Neuro Oncol. 101(2), 311-318 (2010)

73. Karabekir HS, Balci C, Tokyol C. Primary spinal anaplastic ganglioglioma. Pediatr. Neurosurg. 42(6), 374-378 (2006). 
74. Terrier M, Bauchet L, Rigau V et al. Natural course and prognosis of anaplastic gangliogliomas: a multicenter retrospective study of 43 cases from the French Brain Tumor Database. Neuro Oncol. 19(5), 678-688 (2017).

-• Large series of anaplastic ganglioglioma from the French Brain Tumor Database.

75. Mallick S, Benson R, Melgandi W, Giridhar P, Rath GK. Impact of surgery, adjuvant treatment, and other prognostic factors in the management of anaplastic ganglioglioma. Childs Nerv. Syst. 34(6), 1207-1213 (2018).

76. Daumas-Duport C, Scheithauer BW, Chodkiewicz JP, Laws ER Jr, Vedrenne C. Dysembryoplastic neuroepithelial tumor: a surgically curable tumor of young patients with intractable partial seizures. Report of thirty-nine cases. Neurosurgery 23(5), 545-556 (1988).

77. Prayson RA, Estes ML. Dysembryoplastic neuroepithelial tumor. Am. J. Clin. Pathol. 97(3), 398-401 (1992).

78. Honovar M, Janota I. 73 cases of dysembryoplastic neuroepithelial tumor: the range of histological appearance. Brain Pathol. 4, 428-435 (1994).

79. Daumas-Duport C, Varlet P, Bacha S, Beuvon F, Cervera-Pierot P, Chodkiewicz JP. Dysembryoplastic neuroepithelial tumors: nonspecific histological forms - a study of 40 cases. J. Neuro Oncol. 41(3), 267-280 (1999).

80. Bodi I, Selway R, Bannister P et al. Diffuse form of dysembryoplastic neuroepithelial tumour: the histological and immunohistochemical features of a distinct entity showing transition to dysembryoplastic neuroepithelial tumour and ganglioglioma. Neuropathol. Appl. Neurobiol. 38(5), 411-425 (2012).

81. Prayson RA. Diagnostic challenges in the evaluation of chronic epilepsy-related surgical neuropathology. Am. J. Surg. Pathol. 34(5), e1-e13 (2010).

82. Stanescu CR, Varlet P, Beuvon F et al. Dysembryoplastic neuroepithelial tumors: CT, MR findings and imaging follow-up: a study of 53 cases. J. Neuroradiol. 28(4), 230-240 (2001).

83. Chapp C, Padovani L, Scavarda D et al. Dysembryoplastic neuroepithelial tumors share with pleomorphic xanthoastrocytomas and gangliogliomas BRAF(V600E) mutation and expression. Brain Pathol. 23(5), 574-583 (2013).

84. Luzzi S, Elia A, Del Maestro M et al. Dysembryoplastic neuroepithelial tumors: what you need to know. World Neurosurg. 127, 255-265 (2019).

85. Bilginer B, Yalnizoglu D, Soylemezoglu F et al. Surgery for epilepsy in children with dysembryoplastic neuroepithelial tumor: clinical spectrum, seizure outcome, neuroradiology, and pathology. Childs Nerv. Syst. 25(4), 485-491 (2009).

86. Chang EF, Christie C, Sullivan JE et al. Seizure control outcomes after resection of dysembryoplastic neuroepithelial tumor in 50 patients. J. Neurosurg. Pediatr. 5(1), 123-130 (2010).

87. Chassoux F, Rodrigo S, Mellerio C et al. Dysembryoplastic neuroepithelial tumors: an MRI-based scheme for epilepsy surgery. Neurology 79(16), 1699-1707 (2012).

88. Kim DG, Park CK. Central neurocytoma: establishment of the disease entity. Neurosurg. Clin. N. Am. 26(1), 1-4 (2015).

89. Coca S, Moreno M, Martos JA, Rodriguez J, Barcena A, Vaquero J. Neurocytoma of spinalcord. Acta Neuropathol. 87(5), 537-540 (1994).

90. Figarella-Branger D, Soylemezoglu F, Hassoun J. Central neurocytoma. In: Pathology and Genetics of Tumors of the Nervous System. Kleihues P, Cavenee WK (Eds). IARC Press, Lyon, France, 107-109 (2000).

91. Hassoun J, Söylemezoglu F, Gambarelli D, Figarella-Branger D, von Ammon K, Kleihues P. Central neurocytoma: a synopsis of clinical and histological features. Brain Pathol. 3(3), 297-306 (1993).

92. Kim DG, Chi JG, Park SH et al. Intraventricular neurocytoma: clinicopathological analysis of seven cases. J. Neurosurg. 76(5), 759-765 (1992).

93. Maiuri F, Spaziante R, De Caro ML, Cappabianca P, Giamundo A, Iaconetta G. Central neurocytoma: clinico-pathological study of 5 cases and review of the literature. Clin. Neurol. Neurosurg. 97(3), 219-228 (1995).

94. Sharma MC, Deb P, Sharma S, Sarkar C. Neurocytoma: a comprehensive review. Neurosurg. Rev. 29(4), 270-285 (2006).

95. Kulkarni V, Rajshekhar V, Haran RP, Chandi SM. Long-term outcome in patients with central neurocytoma following stereotactic biopsy and radiation therapy. Br. J. Neurosurg. 16(2), 126-132 (2002).

96. Sharma MC, Rathore A, Karak AK, Sarkar C. A study of proliferative markers in central neurocytoma. Pathology 30(4), 355-359 (1998).

97. Sharma MC, Sarkar C, Karak AK, Gaikwad S, Mahapatra AK, Mehta VS. Intraventricular neurocytoma: a clinicopathological study of 20 cases with review of the literature. J. Clin. Neurosci. 6(4), 319-323 (1999).

98. Imber BS, Braunstein SE, Wu FY et al. Clinical outcome and prognostic factors for central neurocytoma: twenty year institutional experience. J. Neurooncol. 126(1), 193-200 (2016).

99. Chen CL, Shen CC, Wang J, Lu CH, Lee HT. Central neurocytoma: a clinical, radiological and pathological study of nine cases. Clin. Neurol. Neurosurg. 110(2), 129-136 (2008).

100. Lee SJ, Bui TT, Chen CHJ et al. Central neurocytoma: a review of clinical management and histopathologic features. Brain Tumor Res. Treat. 4(2), 49-57 (2016). 
101. Zhang D, Wen L, Henning TD et al. Central neurocytoma: clinical, pathological and neuroradiological findings. Clin. Radiol. 61(4), 348-357 (2006).

102. Smith AB, Smirniotopoulos JG, Horkanyne-Szakaly I. From the radiologic pathology archives: intraventricular neoplasms: radiologic-pathologic correlation. Radiographics 33(1), 21-43 (2013).

103. Wang M, Jia D, Shen J, Zhang J, Li G. Clinical and imaging features of central neurocytomas. J. Clin. Neurosci. 20(5), 679-685 (2013).

104. Brandes AA, Amistà P, Gardiman M et al. Chemotherapy in patients with recurrent and progressive central neurocytoma. Cancer 88(1), 169-174 (2000).

105. Johnson MO, Kirkpatrick JP, Patel MP et al. The role of chemotherapy in the treatment of central neurocytoma. CNS Oncol. 8(3), CNS41 (2019).

106. Mrak RE. Malignant neurocytic tumor. Hum. Pathol. 25(8), 747-752 (1994).

107. Enam SA, Rosemblum ML, Ho KL. Neurocytoma in the cerebellum. Case report. J. Neurosurg. 87(1), 100-102 (1997).

108. Giangaspero F, Cenacchi G, Losi L, Cerasoli S, Bisceglia M, Burger PC. Extraventricular neoplasms with neurocytoma features: a clinicopathological study of 11 cases. Am. J. Surg. Pathol. 21(2), 206-212 (1996).

109. Brat DJ, Scheithauer BW, Eberhart CG, Burger PC. Extraventricular neurocytomas. Pathological features and clinical outcome. Am. J. Surg. Pathol. 25(10), 1252-1260 (2001).

110. Gardiman MP, Fassan M, Orvieto E et al. Diffuse leptomeningeal glioneuronal tumors: a new entity? Brain Pathol. 20(2), 361-366 (2010).

111. Rodriguez FJ, Perry A, Rosenblum MK et al. Disseminated oligodendroglial-like leptomeningeal tumor of childhood: a distinctive clinicopathologic entity. Acta Neuropathol. 124(5), 627-641 (2012).

112. Huse JT, Edgar M, Halliday J, Mikolaenko I, Lavi E, Rosenblum MK. Multinodular and vacuolating neuronal tumors of the cerebrum: 10 cases of a distinctive seizure-associated lesion. Brain Pathol. 23(5), 515-524 (2013).

113. Rodriguez FJ, Schniederjan MJ, Nicolaides T, Tihan T, Burger PC, Perry A. High rate of concurrent BRAF-KIAA1549 gene fusion and 1p deletion in disseminated oligodendroglioma- like leptomeningeal neoplasms (DOLN). Acta Neuropathol. 129(4), 609-610 (2015).

114. Lyle MR, Dolia JN, Fratkin J, Nichols TA, Herrington BL. Newly identified characteristics and suggestions for diagnosis and treatment of diffuse leptomeningeal glioneuronal/neuroepithelial tumors: a case report and review of the literature. Child Neurol. Open 2(1), 1-7 (2015).

115. Hakan T, Aker FV. Rosette-forming glioneuronal tumour of the fourth ventricle: case report and review of the literature. Folia Neuropathol. 54(1), 80-87 (2016).

116. Allinson KS, O'Donovan DG, Jena R, Cross JJ, Santarius TS. Rosette-forming glioneuronal tumor with dissemination throughout the ventricular system: a case report. Clin. Neuropathol. 34(2), 64-69 (2015).

117. Allende DS, Prayson RA. The expanding family of glioneuronal tumors. Adv. Anat. Pathol. 16(1), 33-39 (2009).

118. Komori T, Scheithauer BW, Sung JH. Rosette-forming mixed neuronal-glial tumour in the fourth ventricle. Neuropathol. Exp. Neurol. 57, 520 (1998).

119. Scheithauer BW, Silva AI, Ketterling RP, Pula JH, Lininger JF, Krinock MJ. Rosette-forming glioneuronal tumor: report of a chiasmal-optic nerve example in neurofibromatosis type 1: special pathology report. Neurosurgery 64(4), E771-E772 (2009).

120. Anan M, Inoue R, Ishii $\mathrm{K}$ et al. A rosette-forming glioneuronal tumor of the spinal cord: the first case of a rosette-forming glioneuronal tumor originating from the spinal cord. Hum. Pathol. 40(6), 898-901 (2009).

121. Ghosal N, Furtado SV, Hegde AS. Rosette forming glioneuronal tumor pineal gland and tectum: an intraoperative diagnosis on smear preparation. Diagn. Cytopathol. 38(8), 590-593 (2010).

122. Frydenberg E, Laherty R, Rodriguez M, Ow-Yang M, Steel T. A rosette-forming glioneuronal tumour of the pineal gland. J. Clin. Neurosci. 17(10), 1326-1328 (2010).

123. Hakan T, Aker FV. Rosette-forming glioneuronal tumour of the fourth ventricle: case report and review of the literature. Folia Neuropathol. 54(1), 80-87 (2016).

124. Komori T, Scheithauer BW, Hirose T. A rosette-forming glioneuronal tumor of the fourth ventricle: infratentorial form of dysembryoplastic neuroepithelial tumor? Am. J. Surg. Pathol. 26(5), 582-591 (2002).

125. Ellezam B, Theeler BJ, Luthra R, Adesina AM, Aldape KD, Gilbert MR. Recurrent PIK3CA mutations in rosette-forming glioneuronal tumor. Acta Neuropathol. 123(2), 285-287 (2012).

126. Gessi M, Moneim YA, Hammes J et al. FGFR1 mutations in Rosette-forming glioneuronal tumors of the fourth ventricle. J. Neuropathol. Exp. Neurol. 73(6), 580-584 (2014).

127. Jacques TS, Eldridge C, Patel A et al. Mixed glioneuronal tumour of the fourth ventricle with prominent rosette formation. Neuropathol. Appl. Neurobiol. 32(2), 217-220 (2006). 